\title{
Molecular engineering of chromophores for combined second-harmonic and two-photon fluorescence in cellular imaging $\dagger$
}

\author{
Evelien De Meulenaere, ${ }^{a c d}$ Wei-Qiang Chen, ${ }^{b}$ Stijn Van Cleuvenbergen, ${ }^{a}$ Mei-Ling Zheng, ${ }^{b}$ \\ Sotiris Psilodimitrakopoulos, ${ }^{e}$ Rik Paesen, ${ }^{f}$ Jean-Marc Taymans, ${ }^{g}$ Marcel Ameloot,${ }^{f}$ Jos Vanderleyden, ${ }^{c d}$ \\ Pablo Loza-Alvarez, ${ }^{e}$ Xuan-Ming Duan ${ }^{* b}$ and Koen Clays $* a d$
}

Received 7th October 2011, Accepted 22nd January 2012

DOI: $10.1039 / \mathrm{c} 2 \mathrm{sc00771a}$

\begin{abstract}
A series of chromophores with enhanced second- and third-order nonlinear optical properties were engineered for use in combined second-harmonic and two-photon fluorescence microscopy. Electronaccepting moieties imparted nonlinear optical properties to the chromophores. The electron-rich carbazole core served as a template towards one- or two-dimensional chromophores. More efficient acceptor groups (pyridinium, benzazolium, benzothiazolium) on the carbazole donor core resulted in improved second- and third-order nonlinear optical properties. A selection of these chromophores was tested in a cellular environment with a multimodal multiphoton microscope. The structural differences of the chromophores resulted in high selectivity for mitochondria or the nucleus in two-photon fluorescence and ranging from no signal to high selectivity for mitochondria in the SHG channel.
\end{abstract}

\section{Introduction}

Optical microscopy has been since long a truly enabling visualization technique in biological and biomedical sciences. Linear

${ }^{a}$ Department of Chemistry, University of Leuven, Celestijnenlaan 200D, B-3001 Leuven, BELGIUM. E-mail: Koen.Clays@fys.kuleuven.be; evelien.demeulenaere@biw.kuleuven.be; Fax: +32(0)16327982; Tel: $+32(0) 16327508$

${ }^{b}$ Laboratory of Organic NanoPhotonics and Key Laboratory of Functional Crystals and Laser Technology, Technical Institute of Physics and Chemistry, Chinese Academy of Sciences, No.29, Zhongguancun East Road, Beijing, P.R. China 100190. E-mail: chenwq7315@mail.ipc.ac.cn; zhengmeiling@mail.ipc.ac.cn; xmduan@mail.ipc.ac.cn; Fax: +8610 82543597; Tel: +861082543596

${ }^{c}$ Centre for Microbial and Plant Genetics, University of Leuven, G. Geenslaan 1, B-3001 Leuven, Belgium. E-mail: jozef.vanderleyden@biw. kuleuven.be; Fax: +32(0)16321966; Tel: +32(0)16751400

${ }^{d}$ Institute for Nanoscale Physics and Chemistry (INPAC), University of Leuven, Belgium

'ICFO, the Institute of Photonic Sciences, Parc Mediterráneo de la Tecnología, Av. Carl Friedrich Gauss, 3, 08860 Castelldefels (Barcelona), Spain. E-mail: pablo.loza@icfo.es; Fax: (+34) 935534000; Tel: (+34) 935534075

${ }^{f}$ Biomedical Research Institute, Hasselt University, Agoralaan building C, B-3590 Diepenbeek, Belgium. E-mail: marcel.ameloot@uhasselt.be; Fax: +32(0)11269299; Tel: +32(0)11269233

${ }^{g}$ Laboratory for Neurobiology and Gene Therapy, University of Leuven, Belgium. E-mail: Jean-marc.taymans@med.kuleuven.be.; Tel: +32(0) 16332194

$\dagger$ Electronic supplementary information (ESI) available: Additions to Materials and methods section: further details on synthesis and general characterization of the molecules, on nonlinear optical characterization, on the microscope setup used for co-localization, FLIM and TCSPC-SHG imaging. Fig. S1: Two-photon absorption cross section spectra for the compounds studied. See DOI: $10.1039 / \mathrm{c} 2 \mathrm{sc} 00771 \mathrm{a}$ optical microscopy techniques (such as confocal fluorescence), form the basis of modern microscopy. It however suffers from fundamental drawbacks, such as photodamage, photobleaching and low penetration depths, mainly due to the use of short wavelengths for excitation. Nonlinear optical (NLO) microscopy techniques enable us to overcome these problems. This is in part due to the fact that nonlinear excitation normally relies on the use of wavelengths in the near-infrared (NIR) range. At these wavelengths, there is reduced photodamage and increased penetration depth due to a decrease in Rayleigh scattering. In addition, the nonlinear excitation is confined to a focused volume, decreasing phototoxicity and out-of-focus photobleaching. ${ }^{1}$

NLO microscopy can be based on phenomena such as twophoton excited fluorescence (TPEF) and second-harmonic generation (SHG). TPEF is a third-order nonlinear optical effect that results in an optically confined fluorescence emmision. ${ }^{2}$ SHG is a second-order nonlinear optical effect only observed in non-centrosymmetric structures. While chromophores in general can be excited through TPEF (being a third-order nonlinear process, not restricted by any symmetry consideration), noncentrosymmetrical arrangements of non-centrosymmetric chromophores are needed for observing an efficient SHG signal. ${ }^{3-5}$ Although this restricts the number of usable chromophores, it may result in additional structural information about symmetry of the hosting system not provided by single- or two-photon excited fluorescence. ${ }^{6}$ Asymmetrically substituted donoracceptor charge-transfer chromophores have been extensively studied for second-order NLO applications such as electrooptical modulation and terahertz $(\mathrm{THz})$ radiation generation. $\mathrm{THz}$ radiation is generated as the difference-frequency 
component in nonlinear frequency mixing. The trans-4-[4(dimethylamino)- $N$-methylstilbazolium] $p$-tosylate (DAST) molecule is the benchmark molecule and is adopted into a commercially available source for $\mathrm{THz}$ radiation. ${ }^{7}$ The stilbazolium (pyridinium) group not only acts as the electron acceptor group, but also as a polar head group. By elongating the alkyl groups on the donating side, not only a donor-acceptor chargetransfer structure, but also a surface-active amphiphilic molecule is created. In this way, a whole realm of donor-acceptor (or push-pull) molecules combines favorable nonlinear optical properties with interface selectivity. The structure-property relationships relevant between push-pull molecules and nonlinear optical properties have been reviewed. ${ }^{\mathbf{8} 9}$ Design strategies towards chromophores for combined TPEF and SHG should combine function and form requirements. ${ }^{5}$ Function requirements can be alleviated by using strongly polarizable groups such as fluorene ${ }^{5}$ or porphyrin, ${ }^{3}$ while form requirements can be optimized by carbohydrate moieties as the polar head. ${ }^{10}$ The carbazole moiety has been used as a two-dimensional template for engineered chromophores with advanced secondorder NLO properties. ${ }^{11}$ Stilbazolium-substituted carbazole cores have been used in TPEF imaging and in two-photon initiated photopolymerization, both third-order NLO effects, not sensitive to (centro)symmetry. ${ }^{12-14}$ Selectivity for specific cellular targets has been observed and was attributed to different amphiphilic properties or different shapes of the chromophores. ${ }^{15}$

We have now designed and synthesized a library of chromophores, all based on the carbazole core as an electron-rich molecular template for one- (1D) and two-dimensional (2D) chromophores. The resp. mono- or disubstitution of this core with pyridine, nitrophenyl, pyridinium, benzazolium and thiabenzazolium provides a structural diversity which is further enriched by different solubilizing substituents and counter anions for the cationic chromophores. This structural variation leads to variations in linear and nonlinear optical properties, surface activity and toxicity, all highly relevant for the application of combined second-harmonic and two-photon excited fluorescence microscopy.

\section{Materials and methods}

\subsection{Synthesis}

Compound 3,6-bis[2-(pyridin-4-yl)ethenyl]-9-pentylcarbazole (1) was synthesized from 3,6-diiodo-9-pentylcarbazole using Heck coupling reaction. ${ }^{13}$ 3,6-Bis[2-(4-nitrophenyl)ethynyl]-9pentylcarbazole (3) was reported as a photoinitiator, ${ }^{13}$ and using the same method, 3,6-bis[2-(pyridin-4-yl)ethynyl]-9-benzylcarbazole (2) was synthesized. All organic salts were synthesized by Knoevenagel condensation of (substituted) carbazol-3-aldehyde (or 3,6-dialdehyde) with corresponding salts. ${ }^{14}$ The synthetic route was summarized in Scheme 1. Compounds 3, 5, 6, 8 and 9 have been used previously for their two-photon absorption properties only. ${ }^{13,14}$ Compound 3, 4 and $\mathbf{5}$ have also been used as two-photon fluorescent probes in nonlinear microscopy. ${ }^{15}$

Further detailed description of the synthesis and properties of the synthesized molecules can be found in ESI $\dagger$.

\subsection{General physical measurements}

${ }^{1} \mathrm{H}$ and ${ }^{13} \mathrm{C}$ NMR spectra were recorded on a Varian Jemini-300/ Brucker-400 spectrometer and all shifts were referenced to tetramethylsilane. The fine splitting of pyridinyl or phenyl ring patterns is ignored and the signals are reported as simple doublets, with $J$ values referring to the two most intense peaks. Mass spectra were measured on ZAB-HS (Micromass, UK). Elemental analyses were performed on FLASH EA1112 (CE instrument, UK).

The UV-Vis spectra were recorded on a UV-Vis spectrophotometer (UV-2550, Shimadzu) and the fluorescence spectra on a fluorescence spectrophotometer (FL-4500, Hitachi).

\subsection{Nonlinear optical characterization}

The second-order nonlinear optical properties of the molecules were determined by femtosecond hyper-Rayleigh scattering (HRS). ${ }^{16}$ The ionic nature of some of the compounds inherently precluded the use of electric-field-induced second-harmonic generation and necessitated the nonlinear scattering technique that does not rely on the application of a DC electric field. ${ }^{17,18}$ The (multiphoton) fluorescent nature of all the compounds implies the possibility of overestimating the second-order scattering response and hence requires deconvoluting the total signal at the secondharmonic wavelength in the fluorescence and the scattering contribution. This is implemented in the frequency-domain, where at high amplitude-modulation frequencies the time-delayed fluorescence is demodulated and phase-shifted with respect to the immediate scattering. This is the Fourier-transform of the discrimination in the time domain based on the difference in temporal profile between immediate scattering and delayed fluorescence. The measurement procedure was identical to that previously described. ${ }^{19}$ The neutral compounds 1, 2 and 3 were measured in tetrahydrofuran, the ionic compounds 4-11 in methanol.

TPEF spectra were recorded on a commercially available SD2000 spectrometer (Ocean Optics) with the excitation from a mode-locked Ti:sapphire femtosecond laser (Tsunami, SpectraPhysics, tuning range 720-900 nm). Two-photon absorption (TPA) cross sections were obtained by the TPEF method ${ }^{20}$ assuming identical quantum efficiencies $\Phi$ after two-photon excitation as after one-photon excitation. The TPA cross sections are obtained by calibration against fluorescein in aqueous $\mathrm{NaOH}$ solution $(\mathrm{pH}=11)$ at concentrations of $1.0 \times 10^{-4} \mathrm{M}$ for the femtosecond measurement. The samples were dissolved in solvents (methanol, or glycerol or chloroform as indicated) at concentrations ranging from 1.0 to $2.0 \times 10^{-4} \mathrm{M}$. The estimated uncertainty on the retrieved values for the TPA cross section is about $15 \%$. To ensure that the measured signals were solely due to TPA, the dependence of TPEF on the incident intensity was verified in each case to be quadratic. The TPA cross section $\delta$ was calculated on the basis of the following expression:

$$
\delta_{\mathrm{s}}=\delta_{\mathrm{r}} \frac{C_{\mathrm{r}}}{C_{\mathrm{s}}} \frac{n_{\mathrm{r}}}{n_{\mathrm{s}}} \frac{F_{\mathrm{s}}}{F_{\mathrm{r}}} \frac{\Phi_{\mathrm{r}}}{\Phi_{\mathrm{s}}}
$$

with $C$ representing the concentration, $n$ the refractive index of the sample solution, $F$ the integrated area under the TPEF spectrum, and $\Phi$ the fluorescence quantum yield; subscripts s and $\mathrm{r}$ stand for sample and reference, respectively. 


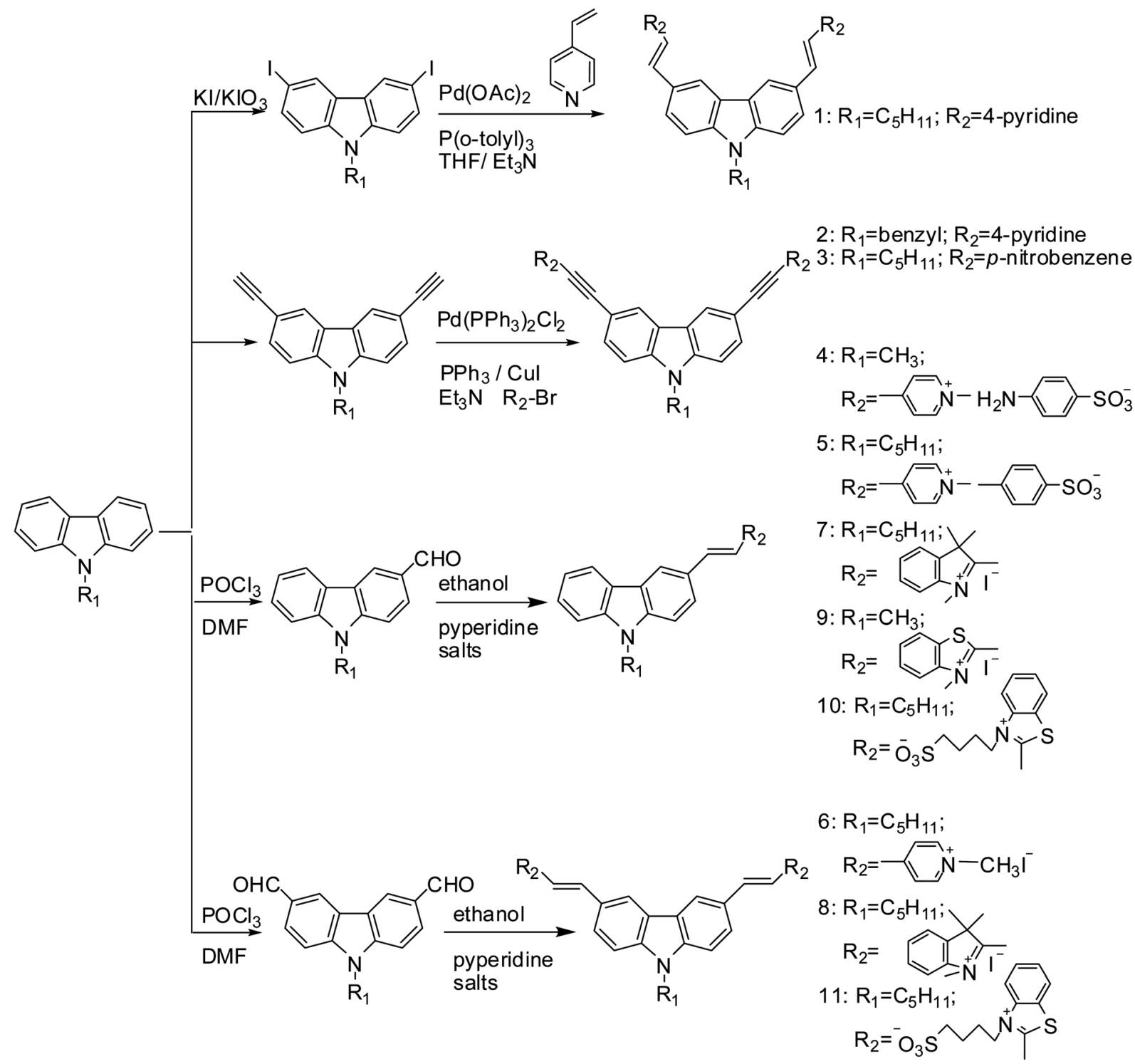

Scheme 1 The synthetic route for compounds 1-11. Compounds 4, 5, 7, 9 and 10 are monosubstituted carbazoles; compounds 1, 2, 3, 6, 8 and 11 are disubstituted.

\subsection{The multimodal (SHG and TPEF) microscopes}

The setup for combined TPEF and SHG microscopy is based on an adapted inverted microscope (TE2000-U, Nikon, Japan) and has been described previously. ${ }^{21}$ The stage of the microscope was installed in an incubator, enabling us to perform experiments at controlled temperatures above room temperature. Because the $\mathrm{CO}_{2}$ concentration in the air was not controlled in the incubator, HEPES-buffered media were used to keep the cells at constant $\mathrm{pH}$.

Co-localization experiments were performed on a confocal microscope Zeiss LSM510 based on an Axiovert 200M inverted microscope and equipped with a META scan box (Zeiss, Jena, Germany) capable of spectral imaging with $10.7 \mathrm{~nm}$ spectral resolution in 32 spectral channels. A maximum of 8 spectral channels can be imaged in one scanning cycle, requiring four scanning cycles for imaging over the entire spectral range of 340 $\mathrm{nm}$. Co-localization was performed with LysoTracker Green, MitoTracker Green and ER-Tracker Green (all trackers by Invitrogen, Germany).

Fluorescence lifetime imaging microscopy (FLIM) was used to discriminate instantaneously occurring SHG (zero lifetime) from TPEF. These experiments were performed on the same setup as the co-localization experiments by using a time-correlated singlephoton counting SPC-830 card obtained from Becker \& Hickl (Berlin, Germany). Detection occurred in transmission through a condenser with a maximal NA of 0.8. Via a dichroic mirror (Di01-R442, Semrock) and a narrow band-pass filter (FF01-395/ 11, Semrock) both the SHG signal and the possibly spectrally overlapping fluorescence signal reach the photon-counting detector (Hamamatsu 7422-P, Berlin, Germany). Data analysis was done using Becker \& Hickl's SPCImage (v. 2.9.5). 


\subsection{Cell toxicity}

The cytotoxicity tests were performed on HeLa cells grown in a 96-well plate. 35000 cells were seeded per well and they were grown for two days in standard culturing conditions. The Cytotoxicity Detection Kit of Roche Applied Science (Germany) was used, which is based on the measurement of lactate dehydrogenase activity in the supernatant. The effect was tested for a range of dye concentrations between $0.5 \mathrm{mM}$ and $0.5 \mathrm{nM}$ in $1 \%$ dimethyl sulfoxide (DMSO) as solvent ( $10 \times$ stock solution), and a range between $2 \mathrm{mM}$ and $2 \mathrm{nM}$ in methanol $(20 \times$ stock solution). The read-out was performed by an EnVision Multilabel Reader (Perkin-Elmer, MA, USA).

\subsection{Sample staining}

For the in cell microscopy experiments, HeLa and MDA-MB435 parental cells (further referred to as 435P cells) were cultured in standard Dulbecco's Modified Eagle Medium (DMEM, Invitrogen, Germany) with 10\% fetal calf serum (FCS) and standard culturing conditions. Before imaging, the cells were carefully washed twice with pre-warmed PBS and new DMEM medium was added. The medium used for imaging lacked phenol red to reduce possible interference with the measurements, but contained HEPES for longer viability of the cells in an environment where only temperature was controlled. Compounds $\mathbf{4}$, 5, 6 and 8 were dissolved in a 1\% DMSO stock solution $(10 \times)$ and because of the low solubility in polar solvents, compounds 7 and 9 were dissolved in $1 \%$ methanol as $10 \times$ stock solution. The solutions were added to the sample on the stage during constant imaging with a final concentration around $10 \mu \mathrm{M}$. Compared to most commercially available dyes, this is a relatively high concentration, but it was acceptable in terms of cytotoxicity of the dyes.

\section{Results}

\subsection{Linear and nonlinear optical characterization}

The variation of the dimensionality of the chromophores is reflected in all of the optical properties. The linear absorption spectra for the disubstituted derivates $1,2,3,6,8$ and 11 exhibit a clear band splitting, being a typical characteristic of electronic interaction between dimers (see Fig. 1 and Table 1, columns 2, 4 and 5). The relative oscillator strength for the two bands appears to vary with the acceptor (weak high-energy shoulder for the benzazolium (8), clear shoulder for the pyridinium (6), roughly equal strength for the benzathiazolium (11) and inversion of the relative strength for the weak pyridine acceptors in $\mathbf{1}$ and $\mathbf{2}$, although it is hard to derive conclusions without the monosubstituted (1D) derivatives). The overall nonlinear response for the disubstituted derivatives, as expressed both in the $\beta_{\mathrm{HRS}}$, and in the $\delta_{800}$ (Table 1, columns 6, 7 and 14) is always significantly larger and often approximately twice the overall response for the monosubstituted analogue (compare 6 with 5; 8 with 7; and 11 with 10). Another striking observation is the significantly and consistently lower value for the depolarization ratio $\rho$ for the disubstituted derivatives $\mathbf{3}, \mathbf{6}, \mathbf{8}$ and $\mathbf{1 1}$, all below 1.7, while for the monosubstituted derivatives $\mathbf{4}, \mathbf{5}, \mathbf{7}, \mathbf{9}$ and $\mathbf{1 0} \rho$ is at least 2.6 and up to 3.6 (see Table 1, column 8). Such a high depolarization ratio is consistent with the largely $1 \mathrm{D}$ nature of the chargetransfer and has been interpreted towards a single major dipolar hyperpolarizability component $\beta_{z z z, 800}$ and the static $\beta_{z z z, \mathrm{o}}$ after correction for the resonance enhancement. The lower value for the depolarization ratio for the disubstituted V-shaped compounds corroborates their 2D nature. From the two observables in HRS (i.e. the total intensity and the depolarization ratio, $\rho$ ) it is possible to derive the two non-zero tensor components assuming approximate $C_{2 \mathrm{v}}$ symmetry, $\beta_{z z z}$ and $\beta_{x z z}$. This interpretation in terms of tensor components (with and without resonance enhancement) is provided in columns 8-13 of Table 1. The last column provides the (dynamic, not corrected for resonance enhancement) two-photon absorption coefficients, $\delta_{800}$, in units of Goeppert-Mayer (GM). The TPA spectra are provided in Fig. S1 (ESI $\dagger$ ). There are a number of experimental observations that confirm the potential of well-designed 1D and 2D carbazole-based chromophores for combined second- and third-order nonlinear optical (imaging) applications. A first observation (see Table 1, rows 1 and 2) is that the effective donor$\pi$-donor design (compounds $\mathbf{1}$ and $\mathbf{2}$ ) that has been proposed to suffice to induce third-order NLO properties ${ }^{22}$ such as TPEF for high-resolution imaging, is not leading to a detectable secondorder, nor third-order NLO response. The largely symmetric design in terms of electron-donating or withdrawing properties is also reflected in the shortest wavelength of maximal absorption. There is a subtle effect of the nature of the bridge between the carbazole and the pyridine moiety (ethenilic in $\mathbf{1}$ or ethynilic in 2), or of the substituent on the carbazole nitrogen, but this is only experimentally discernible in linear optics (UV-Vis absorption and fluorescence). There is a detectable signal at the secondharmonic wavelength, but the high-frequency fluorescence demodulation approach is clearly showing that the small signal is originating from TPEF. It is not possible to conclude that there is a non-zero second-order nonlinear response. Hence, no values for first hyperpolarizability $\beta_{\mathrm{HRS}, 800}$ (nor depolarization ratio, vide infra) have been deduced for compounds $\mathbf{1}$ and $\mathbf{2}$. Even though a certain TPEF contribution to the HRS signal was detected, no TPA cross section $\delta_{800}$ could be reliably derived at $800 \mathrm{~nm}$ either.

Already upon replacing pyridine by nitrophenyl (compound 3), with a nitro group acting as an electron withdrawing group, there is a pronounced effect on the optical properties. The charge-transfer that is induced between the carbazole donor moiety and the nitro acceptor not only shifts the wavelength of maximum absorption and emission to a longer wavelength (lower transition energy), also the second- and third-order NLO response is no longer negligible and is significantly enhanced by two-photon resonance. The dynamic value for the first hyperpolarizability, $\beta_{\mathrm{HRS}, 800}$, is indeed considerable, but strongly affected by the electronic resonance close to the second-harmonic wavelength (two-photon enhancement). A rough estimate of this effect can be obtained from the two-level model. ${ }^{23,24}$ Without the effect of damping, the effect may be overestimated, but the resulting static value $\beta_{\mathrm{HRS}, \mathrm{o}}$ of compound $\mathbf{3}$ is the smallest of all the compounds reported in this work.

Upon replacing the nitro acceptor group by the stronger pyridinium group (compounds 4, 5 and $\mathbf{6}$ ), we observe in linear optics a further lowering of the transition energy for the charge transfer state and a concomitant increase in the static first 

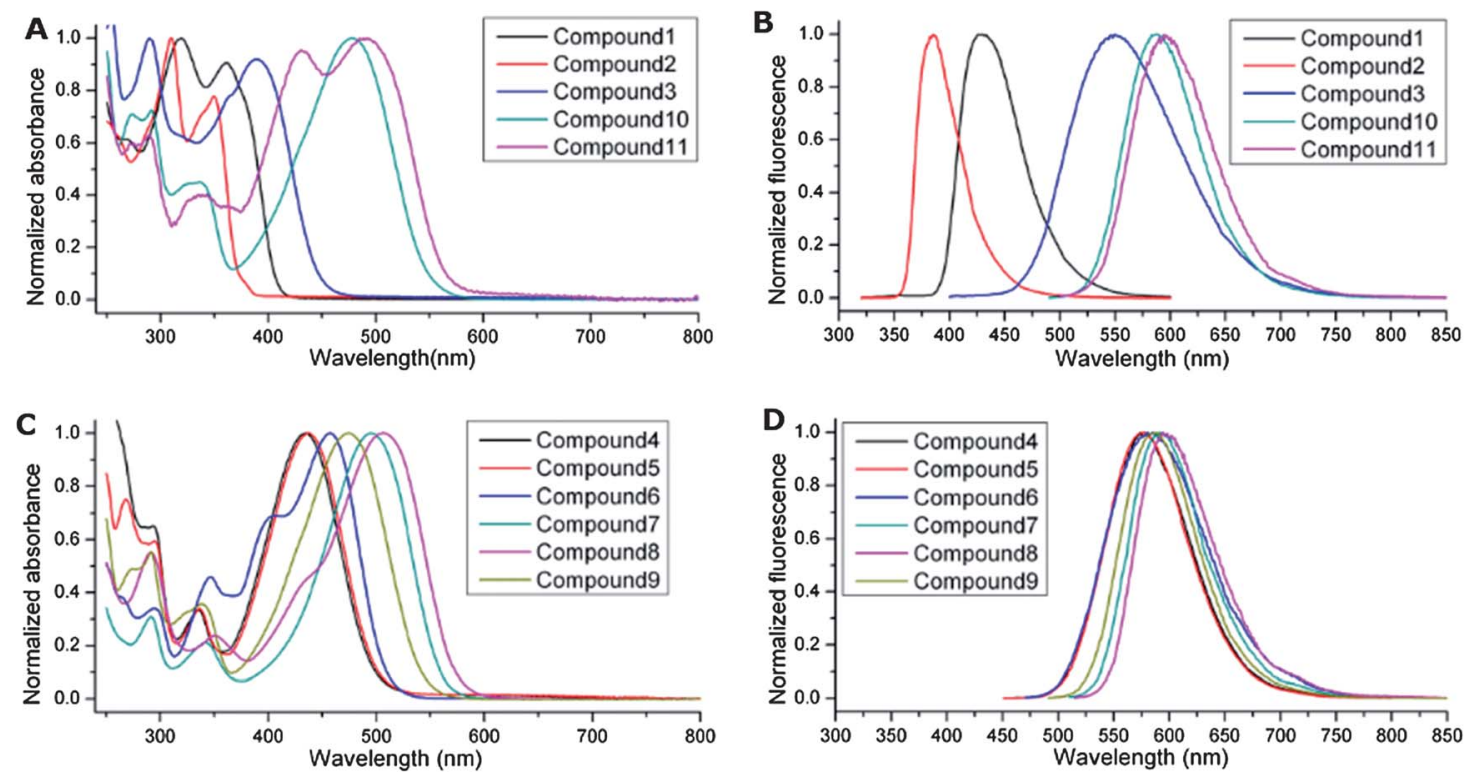

Fig. 1 Absorbance and fluorescence spectra of all 11 compounds. Graphs A and C present absorbance spectra, graphs B and D the fluorescence spectra. Graphs $\mathrm{C}$ and $\mathrm{D}$ show the spectra of the compounds that were selected for the microscopy experiments.

hyperpolarizability $\beta_{\mathrm{HRS}, \mathrm{o}}$. The dynamic properties at $800 \mathrm{~nm}$ are less resonantly enhanced, resulting in lower values for $\beta_{\mathrm{HRS}, 800}$ and $\delta_{800}$. Essentially identical values for the optical properties have been obtained for compounds $\mathbf{4}$ and $\mathbf{5}$ with different counter anions, confirming the conjecture that the NLO properties of stilbazolium-derived chromophores find their origin entirely in the cationic chromophore and are not traced to the counter anion. ${ }^{25}$
Further strengthening the accepting capacity by replacing the pyridinium group by a benzazolium (compounds 7 and $\mathbf{8}$ ) or a benzathiazolium group (compounds 9, 10 and 11) results in a concomitant further decrease of the transition energy and increase in static hyperpolarizability. As a result, some of the largest values for $\beta_{\mathrm{HRS}, \mathrm{o}}$ and $\delta_{800}$ are obtained for these compounds, keeping in mind that the dynamic hyperpolarizability $\beta_{\mathrm{HRS}, 800}$ is affected by the dimensionality

Table 1 Linear and nonlinear optical properties of carbazole-derived chromophores: $\lambda_{\text {abs,max }}$, wavelength of maximal absorption (two values indicate the two bands, if applicable); $\lambda_{\mathrm{em} \text { max }}$, wavelength of maximal emission; $\Delta E$, energy splitting between the two bands (if applicable); $\varepsilon$, extinction coefficient (two values relate to the two bands, if applicable); $\beta_{\mathrm{HRS}, 800}$, total HRS response (total intensity, not related to specific tensor components, dynamic value at $800 \mathrm{~nm}) ; \beta_{\mathrm{HRS}, \mathrm{o}}$, total HRS response, corrected for resonance enhancement; $\rho$, HRS depolarization ratio; $k^{-1}=\beta_{z z} / \beta_{x z z}$, derived ratio between major tensor components; $\beta_{z z z, 800}$, diagonal hyperpolarizability tensor component, dynamic $800 \mathrm{~nm}$ value; $\beta_{z z z, \mathrm{o}}$, diagonal hyperpolarizability tensor component, static value; $\beta_{\mathrm{xzz}, 800}$, off-diagonal hyperpolarizability tensor component, dynamic $800 \mathrm{~nm}$ value; $\beta_{x z z, \mathrm{o}}$, off-diagonal hyperpolarizability tensor component, static value; $\phi$, fluorescence quantum yield; $\delta_{800}$, two-photon absorption cross-section at $800 \mathrm{~nm}$

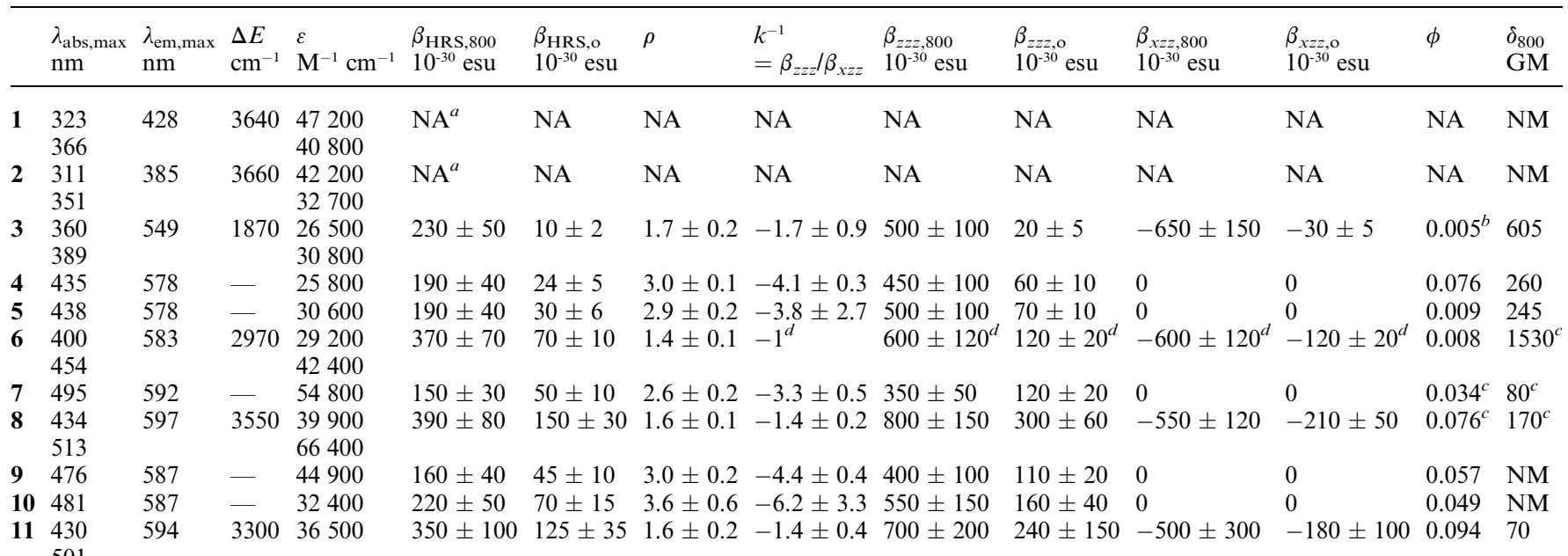

${ }^{a}$ Only TPEF contribution to HRS signal, no value available. ${ }^{b}$ Determined in chloroform. ${ }^{c}$ Determined in glycerol. ${ }^{d}$ Derived from the theoretically lowest possible value for $\rho$ of 1.5. NM: not measurable due to weak TPEF signal. 
(mono- or disubstituted) and the varying shifts between the second-harmonic and the resonance wavelength. However, for any optical application, the actual wavelength of the light used is ultimately determining the amplitude of the (nonlinear) optical response. Therefore, the dynamic values for the optical parameters $\left(\beta_{\mathrm{HRS}, 800}\right.$ and $\left.\delta_{800}\right)$ are what is really important in assessing the suitability of these chromophores for nonlinear imaging with a titanium-sapphire laser.

The addition of solubilizing groups (pentyl, benzyl or methyl on the tertiary carbazole nitrogen; and butylsulfonate vs. methyl on the quaternary thiabenzazolium nitrogen) has no effect on the linear and nonlinear optical properties, within the estimated uncertainties. However, the solubility of the compounds in polar solvents is an important feature for the use in microscopy of biological samples.

\subsection{Cell toxicity}

An important issue with extrinsic staining of live samples is the toxicity of the marker compound. It is therefore advised to keep the concentration of the dye as low as possible. However, this is always a trade-off between toxicity and visibility of the dye. In this early stage of characterization of the dye, we used reasonably high concentrations in order to increase the likelihood of detecting the markers in the SHG channel of the microscope. We chose the concentration based on a cytotoxicity test performed on HeLa cells. The tested range of concentrations was a 10 -fold dilution series starting near the maximum solubility in $1 \%$ DMSO and a two-fold higher concentration in methanol. The toxicity was comparable for most of the dyes, with slightly higher values for compound 7 (results not shown). For incubation times as long as $3 \mathrm{~h}$, which is longer than the maximal observation time, the toxicity read-outs were comparable to the negative control for all except the highest concentration, both for the aqueous (50 $\mu \mathrm{M}$ final concentration) and the methanol solutions ( $0.1 \mathrm{mM}$ final concentration). For these concentrations, the effect on the cells was already visible after $1 \mathrm{~h}$.

\subsection{Combined SHG and TPEF microscopy}

As a reference, unstained live cells were imaged for $1 \mathrm{~h}$ on the microscope stage with constant exposure to the laser. We observed multiphoton excited autofluorescence but no SHG for the HeLa cells, while we recorded no signal at all for the 435P cells in either of the channels. During these experiments, we also monitored the shape of the cell to confirm their viability in the microscope incubator. Since the cells did not change shape nor autofluorescence intensity and remained attached to the glass surface, we assumed the imaging conditions were acceptable.

In imaging experiments earlier reported using compounds 5 and the cation parts of compounds $\mathbf{4}$ and $\mathbf{6},{ }^{15}$ the staining was done by $30 \mathrm{~min}$ incubation with $1 \mu \mathrm{M}$ concentrations of the dyes. The dyes were dissolved in water or in low DMSO concentrations. To increase the chance of observing any SHG signal, concentrations as high as the cytotoxicity experiments would allow were used. For compounds 4, 5, 6 and $\mathbf{8}$, we diluted the dyes to $0.1 \mathrm{mM}$ in $1 \% \mathrm{DMSO}$, while for compounds 7 and 9 the solubility in DMSO was still too low for our purposes. We therefore dissolved these dyes in a $1 \%$ methanol solution. We consequently diluted these stock solutions 10 -fold in the imaging medium. Besides prestaining the samples, we also added the dye during the imaging process. This resulted in a more stable SHG signal for some of the dyes and offered us an opportunity to study the staining dynamics, for both the TPEF and the SHG emitted signals.

The results of the combined TPEF and SHG imaging are presented in Fig. 2, where TPEF is represented in red and SHG in green. The figure gives an overview of representative results for all of the selected compounds. Most of the dyes are represented in HeLa cells, while compounds 6 (Fig. 2C) and 7 (Fig. 2D) are represented in $435 \mathrm{P}$ cells because the contrast was much higher. The dyes are excessively present in the samples, resulting in a certain background signal in the cytosol that is only observed in the TPEF channel. To pinpoint the origin of the TPEF and SHG signals, we performed co-localization experiments with LysoTracker Green, MitoTracker Green and ER-Tracker Green. For four of the six tested compounds (compounds 4, 5, 7 and 8), TPEF only shows up in the mitochondria, while compound $\mathbf{6}$ seems to be the only one targeting the nucleus instead of the mitochondria, although compound 9 also exhibits a significant TPEF contribution in the nucleus besides the general mitochondrial targeting.

The recorded SHG images however, turned out to be more exciting than the observations in the TPEF channel. Not only did the respective dyes show up in different locations, we also observed signal originating from locations other than what the TPEF would suggest. While - according to the observations in TPEF - compounds $\mathbf{4}, \mathbf{5}, \mathbf{7}, \mathbf{8}$ and $\mathbf{9}$ are targeted to the mitochondria, we only find a high spatial correlation with the SHG signal in the cells stained with compound 4. In terms of SHG, compounds 5, 7 and 9 behaved comparatively similarly to each other, all revealing plasma membranes and certain organelles or vesicles. For compound 7 the SHG signal is not observed during the healthy life span of the dyes, but it only appears shortly before the onset of observable cell death. We therefore assume that we observe the "blebbing" process of the apoptotic cells and that the organelles that appear are vacuoles, about to be ejected from the cell (visible in Fig. 2D).

Compounds $\mathbf{6}$ and $\mathbf{8}$ are disubstituted carbazoles and therefore stand out from the list in several of their properties, also in microscopy. Compound $\mathbf{6}$ distinguishes itself by exhibiting a remarkably different TPEF pattern, targeting the nucleus and mitochondria, leading to the hypothesis that this dye can intercalate with DNA. The SHG signal however, does not originate in the nucleus or the mitochondria. Compound $\mathbf{8}$, the other disubstituted compound tested with SHG imaging, was targeted to the mitochondria as well (in TPEF), but never showed any signal in the SHG channel at all. An overview of some important molecular features and localization in TPEF and SHG channels is summarized in Table 2.

Fig. 3 displays the dependence of the SHG response to the orientation of the polarization of the fundamental laser. SHG as an optical phenomenon is extremely sensitive to the relative orientation of the polarization of the laser compared to the orientation of the (induced) dipole. This dependency is also used in fluorescence anisotropy measurements, ${ }^{26}$ but in SHG this effect is immediately visible in our images, even without the use of an analyser in the setup. The phenomenon is very simple: when 

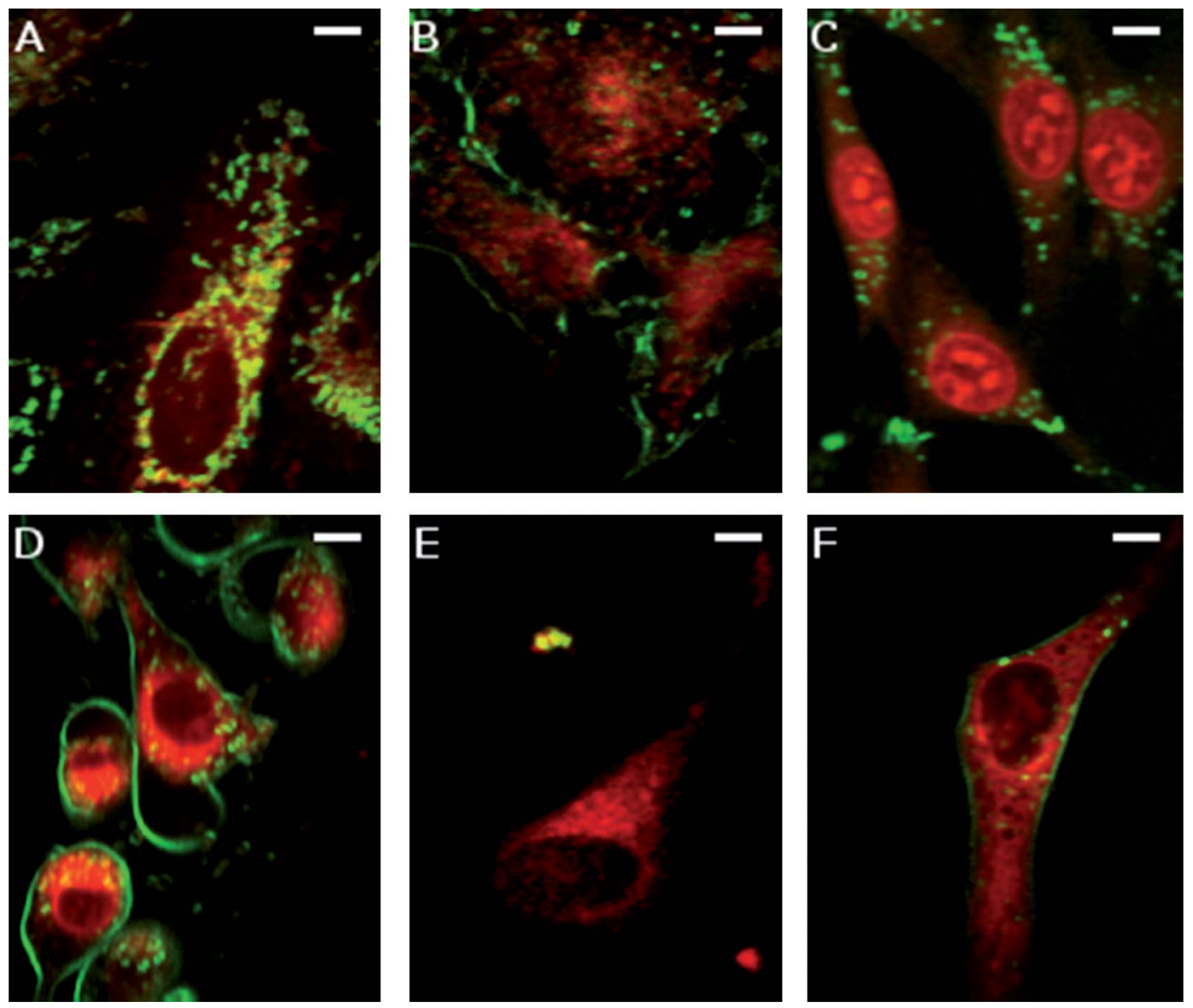

Fig. 2 Results of combined TPEF (red) and SHG (green) imaging in 435P and HeLa cells. We show compounds 4 (A), 5 (B), 8 (E) and 9 (F) in HeLa cells, and compounds 6 (C) and 7 (D) in 435P cells. The white bars indicate $10 \mu \mathrm{m}$.

the linear polarization of the laser is parallel to the induced dipole, the SHG signal will be maximal, while it will be zero when the induced dipole is in the plane perpendicular to the electric field vector of the linearly polarized laser light. The effect is enhanced by the quick decrease of SHG intensity in locations with randomization and centrosymmetrical arrangements of the chromophores, creating a high contrast. Since SHG occurs instantaneously, it has no lifetime, and therefore no effect can be seen due to the dynamics of the molecules, which is the case for fluorescence anisotropy measurements.
Fig. 3A, B and C show images taken with the orientation of the laser polarization advancing in steps of 60 degrees, visualized by the white arrows. The strong dependence on the orientation of the laser polarization was confirmed by the apparent SHG intensity change along the membranes as we gradually changed the orientation of the laser polarization. Fig. 3D is an overlay of the former three images in the blue, the red and the green channel, respectively, not to be confused with color-scaled images displaying calculated orientations of the dyes in the membrane. ${ }^{27}$ The sample in Fig. 3 was stained with compound $\mathbf{5}$,

Table 2 Summary of some important characteristics of the dyes, related to molecular structure and visibility of cellular structures in TPEF or SHG channel

\begin{tabular}{|c|c|c|c|c|c|c|c|c|c|}
\hline \multicolumn{4}{|c|}{ Molecular properties } & \multicolumn{3}{|c|}{ Visible in TPEF? } & \multicolumn{3}{|c|}{ Visible in SHG channel? } \\
\hline & Completely planar? $^{a}$ & $1 \mathrm{D} / 2 \mathrm{D}$ & Hydrophobic tail & Mitochondria & Nucleus & Plasma membrane & Mitochondria & Plasma membrane & Organelles \\
\hline 4 & Yes & $1 \mathrm{D}$ & $\mathrm{CH}_{3}$ & Yes & Weak & & Yes & & \\
\hline 5 & Yes & 1D & $\mathrm{C}_{5} \mathrm{H}_{11}$ & Yes & & Weak & & Yes & Yes \\
\hline 6 & Yes & $2 \mathrm{D}$ & $\mathrm{C}_{5} \mathrm{H}_{11}$ & Yes & Yes & Weak & & & Yes \\
\hline 7 & No & 1D & $\mathrm{C}_{5} \mathrm{H}_{11}$ & Yes & & Late & & Yes & Yes \\
\hline 8 & No & $2 \mathrm{D}$ & $\mathrm{C}_{5} \mathrm{H}_{11}$ & Yes & & & & & \\
\hline 9 & Yes & $1 \mathrm{D}$ & $\mathrm{CH}_{3}$ & Yes & Weak & Weak & & Yes & Yes \\
\hline
\end{tabular}



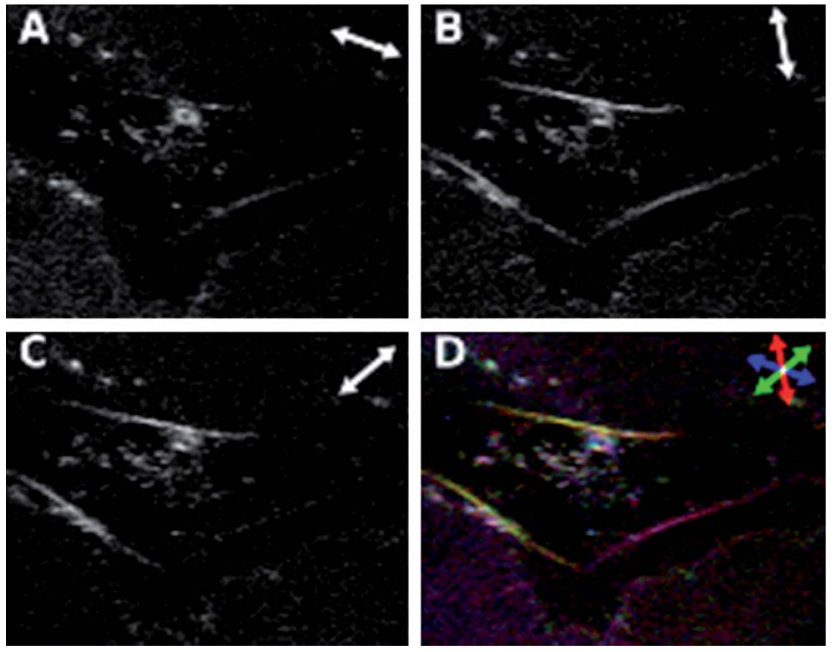

Fig. 3 Polarization-dependence of a HeLa cell stained with compound 5. The white arrows show the orientation of the linearly polarized incident laser beam (A, B, C). Section D shows an overlay of the other three images, respectively in the blue, the red and the green channel. The length of the arrows represents $20 \mu \mathrm{m}$ in the image.

but this behavior was also observed when we applied different orientations of the polarization of the laser light to samples stained with compounds $\mathbf{7}$ and $\mathbf{9}$. It is however unclear as to why the molecules do not create centrosymmetry by residing in both leaflets of the membrane, or why the signal does not suffer from this (see later in discussion).

\section{Discussion}

\subsection{Linear and nonlinear optical characterization}

The second-order NLO response of mono- and dinitrocarbazoles 3-nitro-9-ethylcarbazole and 3,6-dinitro-9-ethylcarbazole has been previously studied. ${ }^{9}$ Nonzero, but significantly smaller nonlinearities had been obtained. However, the difference in dimensionality was also observed, starting from a lower value for the depolarization ratio and resulting in more significant off-diagonal tensor elements for the disubstituted derivative. For the derivatives studied here, with the longer conjugated bridge and the stronger acceptors, hyperpolarizabilities of at least one order of magnitude higher have been observed.

Tuning the magnitude of the linear and the second- and thirdorder NLO responses is often rationalized in terms of donor and acceptor strength, and length of the $\pi$-conjugation bridge. Here, we did not vary the length of the bridge, or the nature of the donor (the carbazole core). The acceptor strength is varied from absent (pyridine, compounds $\mathbf{1}$ and 2) over moderate (nitrophenyl in compound 3) to strong (pyridinium, 4-6) and strongest (benzazolium in compounds $\mathbf{7}$ and $\mathbf{8}$, and benzathiazolium in 9-11). The experimental values for the dynamic first hyperpolarizabilities at $800 \mathrm{~nm}$ are not only a function of this strength, but also of the wavelength, through the single- and two-photon resonance enhancement. ${ }^{24}$ The result is that, although pyridinium derivatives exhibit lower transition energies than nitrophenyl, the latter shows the larger dynamic hyperpolarizability. Upon correcting for this resonance enhancement though, the static hyperpolarizability becomes the smallest of the non-zero values (zero for the pyridine derivatives).

The disubstituted variants can not simply be described as two non-interacting monosubstituted analogues. For two non-interacting monomeric model compounds, the only difference in the electronic absorption spectra should be a doubling of the extinction coefficient. This clearly is not the case here. Since the carbazole moiety is shared as donor for the two branches each with the accepting unit at their end, there must be an electronic effect that has its influence on the linear optical properties. This is apparent as the band splitting of the main absorption band in the linear absorption spectrum. ${ }^{28}$ Therefore we analyzed our secondorder NLO results not in terms of the vector sum of the vector parts of two separate hyperpolarizability tensors, but as a single hyperpolarizability tensor with more than just the single dominating diagonal tensor element. Since we have two independent observables (HRS intensity and depolarization ratio) we can deduce two independent non-zero tensor elements. The analysis shows that the ratio between the diagonal and off-diagonal tensor element $\left(k^{-1}\right.$ in Table 1$)$ is between $6 \pm 3$ and $3.3 \pm 0.5$ for the monosubstituted derivatives (corroborating a largely 1D hyperpolarizability tensor) and between 1 and $1.7 \pm 0.8$ for the disubstituted (indicating substantial off-diagonal contributions). This allows us to deduce actual values for the on- and off-diagonal values of the hyperpolarizability tensor for the disubstituted carbazole derivatives.

The values for the dynamic TPA cross section, $\delta_{800}$ (see last column in Table 1 and Fig. S1, ESI $\dagger$ ) can be rationalized entirely in terms of resonance enhancement and molecular footprint. The larger value for $\delta_{800}$ observed for the disubstituted carbazole derivatives traces its origin directly to the larger two-dimensional $\pi$-conjugated system, inherently related to linear and nonlinear absorption cross sections. Note that the (linear) extinction coefficient $\varepsilon$ in column 5 is not a direct measure of the total absorption cross section for linear absorption, as this single numerical value does not reflect the band splitting and the resulting stronger total oscillator strength from integrating over the larger spectral width of the transition. The electron-donating or -accepting strengths are not correlated with the symmetry-insensitive third-order NLO property of TPA. This is strikingly exemplified in compound $\mathbf{3}$, which shows the smallest second-order NLO response of the disubstituted derivatives (both before and after correction for resonance), but the second largest value for $\delta_{800}$. This already points to the inherent differences between a second-order and a third-order NLO effect, here observed and analyzed at the level of molecular symmetry: TPA is not sensitive to molecular symmetry, in contrast to HRS, the latter being of a second-order nature. For applications in imaging of targets stained with chromophores, similar symmetry arguments lead to the important difference in information that can be gained from SHG imaging vs. TPEF imaging. When combined with the resonance enhancement effect, the larger footprint of disubstituted compound $\mathbf{6}$ and its resonance features around the second-harmonic wavelength result in the largest TPA cross-section when measured at 800 nm (see Table 1 and Fig. S1, ESI $\dagger$ ). 


\subsection{Combined SHG and TPEF microscopy}

Compound $\mathbf{5}$ and the cationic parts of compounds $\mathbf{4}$ and $\mathbf{6}$ have been previously imaged in HeLa cells using TPEF microscopy only. ${ }^{15}$ While compounds 4 and 5 were observed in the mitochondria, compound $\mathbf{6}$ was directed to the nucleus. We have already reported on the combined TPEF and SHG imaging of compound $\mathbf{5},{ }^{29}$ where the SHG signal was observed in vesicles, presumed to be lysozomes. Apart from these previously described markers, we also imaged compounds 7, 8 and 9. The selection of the dyes used for SHG microscopy was based on three criteria. First, the hyperpolarizability of compounds 1 and 2 was not measurable in the HRS experiments. Second and more importantly, compounds $\mathbf{1}, \mathbf{2}$ and $\mathbf{3}$ were omitted due to the expected fluorescence contribution at $405 \mathrm{~nm}$, as our laser was working at the central wavelength of $810 \mathrm{~nm}$. Although it is possible to filter out SHG from a fluorescence background based on fluorescence lifetimes (Time Correlated Single Photon Counting (TCSPC) microscopy), ${ }^{30}$ or to discern SHG from TPEF by polarization-sensitive SHG microscopy, ${ }^{31}$ it is much more convenient to use markers that exhibit no fluorescence interference in the SHG channel. As most commercially available two-photon systems operate at optimal performance between 760 and $900 \mathrm{~nm}$, it is not advisable to use a dye that starts fluorescing at $350 \mathrm{~nm}$. The third selection rule was the solubility of the compound in aqueous solvents, which was very low for compounds 10 and 11. This way we decided that compounds 4-9 seemed fit for SHG imaging of live cells at a fundamental wavelength of $810 \mathrm{~nm}$.

The expectations of the visibility of exogenous dyes in the SHG channel of our microscope were concentrated on a small selection of cellular structures. These comprise the plasma membrane, because the structure of membranes inherently forces the molecules in a specific, well-ordered orientation. The visibility of membranes using SHG has been previously shown in studies using cells or synthetic vesicles. ${ }^{3,32,33}$ Secondly, since SHG is not only sensitive to high structural non-centrosymmetrical order in a parallel fashion, but also to chirality, ${ }^{34-36}$ a chiral structure such as (alpha-helical) DNA is also a candidate for SHG response, certainly because compound $\mathbf{6}$ had been previously imaged inside the nucleus using TPEF. In eukaryotic cells, the DNA is ordered in several levels of chiral structures, such as nucleosomes and solenoids, resulting in chiral structures much bigger than the alpha helix of naked DNA. This can have a beneficial effect, but can also work to our disadvantage, as the final helical structures by themselves are not always arranged in an orderly manner. We were careful in predicting the visibility of intracellular structures with $\mathrm{SHG}$, as most of these structures are smaller than the wavelength we use. The introduction of the markers in intracellular vesicles can therefore result in centrosymmetry or out-of-phase responses. Finally, we were also hoping to be able to follow the penetration of the molecules into the plasma membrane during staining, because the planar molecules can only enter the membrane parallel to the fatty acid chains.

The results of the combined TPEF and SHG imaging were therefore very surprising. While most of the dyes show fluorescence in the mitochondria, we observed different behavior for the respective compounds in SHG. The two extremes were compound 4 on one hand, exhibiting a high spatial correlation of the SHG with the TPEF signal, highlighting the mitochondria, and compound $\mathbf{8}$ on the other hand, with a nice TPEF contrast but no SHG at all. This observation confirms very nicely that a high $\beta$ is not the only criterion of a good cellular SHG marker. It is therefore meaningless to discuss the numerical data from Table 1 with regard to the imaging experiments. The other four markers $(5,6,7,9)$ show up in different locations in the two channels. Furthermore, the SHG signal of several of the dyes was observed in the plasma membrane, as we expected, but never while the molecules were entering the cell during the staining process. On the contrary, the SHG signal only appeared later in the experiment. This suggests that the penetration of the molecules through the membranes does not happen in a well-ordered way. The orientation of the markers is most probably in line with the fatty acid chains, but we assume that either the orientation is too chaotic, or a centrosymmetric ordering of the molecules occurs because the direction in which the molecules enter the membrane varies. It is therefore likely that the SHG signal appears as soon as the orientation of the markers inside or connected to the membrane assume exactly the same orientation. For compound 7 it took even longer before the SHG signal appeared, as we had to wait until near cell death. At that moment, the integrity of the membranes changes, possibly allowing the relatively large and not entirely planar dye to enter between the fatty acid chains. This property could make compound 7 a marker for approaching cell death.

Although membranes are ideal scaffolds to create molecular order, it is not straightforward that they can also serve this purpose for SHG, which cannot detect centrosymmetrical order. Biological membranes are usually lipid bilayers, inherently inactive in SHG. Molecules that can penetrate these structures, can do so in the same manner from both sides, only creating more centrosymmetry. The fact that we can observe the membranes, even though fluorescence indicates that the molecules can enter the membranes from both sides, proves that there is a net directionality of the orientation of the (induced) dipoles. This can be explained either by slow dynamics of the distribution of the chromophores between the two leaflets of the membranes, ${ }^{1}$ or the molecular orientation might be driven by a membrane potential.

Apart from the plasma membrane, we were also able to visualize intracellular structures other than those visible in the TPEF channel. As previously mentioned, the SHG and TPEF images of compound 4 show a very nice spatial correlation in the mitochondria, pointing towards possible TPEF leakage in the small spectral region of the $406 / 15 \mathrm{~nm}$ filter. Taking into account that the resolution of the setup is too low to check for polarization dependency in this sample, the nature of this signal was doublechecked by means of TCSPC-SHG, ${ }^{30}$ based on fluorescence lifetime imaging microscopy (FLIM), and by spectral filtering (lambda-stack) to confirm the observation of $\mathrm{SHG}$ and not TPEF leakage.

\subsection{Structure-function analysis for cellular imaging}

First of all, all compounds in this study have a hydrophobic character, leading to a strong tendency to reside inside membranes. Because of the solubility restraints, all compounds 
selected for microscopy are cationic, leaving some hydrophilic properties as well. Our previous characterisation of carbazolebased cyanine dyes based on TPEF microscopy showed that the cellular targeting of the dyes can be adapted by changing the molecular symmetry (or dimensionality) and the length of the hydrophobic chain connected to the carbazole moiety. ${ }^{15} \mathrm{We}$ concluded from those experiments that 1D compounds with a long tail $\left(\mathrm{C}_{5} \mathrm{H}_{11}\right)$ are directed to mitochondria, $1 \mathrm{D}$ compounds with a short tail $\left(\mathrm{CH}_{3}\right)$ to mitochondria and the nucleus. The $2 \mathrm{D}$ compounds target only the nucleus when they have a short tail, and with a long tail, they go to both the nucleus and mitochondria. In both cases, the molecules with a long tail are observed in the plasma membrane. Our recent observations are mostly consistent with these rules, but we find a few exceptions in the TPEF channel, and some new targets in the SHG channel that are hardly visible in TPEF (see Table 2).

In the TPEF channel, all except compound $\mathbf{6}$ are primarily visible in mitochondria, suggesting that the molecular properties result in high affinity for this cellular location. Explaining the targeting of mitochondria is a difficult matter, as several dyes are actively taken up by these organelles. It is also known that cationic fluorescent dyes, such as Rhodamine 123, can stain mitochondria selectively because they might be attracted to mitochondria by the relatively high negative electric potential across the mitochondrial membrane. ${ }^{37}$ We don't have the possibility to determine whether the marker molecules are residing merely in the membranes of the mitochondria, in their lumen, or whether they are visible in the mitochondria because of active transport.

Compound $\mathbf{6}$ is found in the nucleus, with a particularly higher concentration in the nuclear membrane and the less condensed parts of the nucleus (nucleoli). It can also be observed in mitochondrial DNA, as the signal is distinguishable from the autofluorescence background. Previous research also reports that carbazole-based cyanine derivatives may stain the nucleus. $^{38,39}$ A closer look at the structure of compound 6 indeed tells us that it is possible for this planar and bent molecule to either intercalate with DNA, perhaps involving a stronger interaction than with membrane lipids, e.g. interaction with G-quadruplex DNA structures. ${ }^{38,39}$ Compound 8 however, the other 2D compound in our selection, contains two sites extruding from the planar bulk of the molecule, obviously preventing DNA intercalation.

In the SHG channel, not only the targeting, but also the molecular ordering and orientation will define where the dye will cause contrast in the imaging process. This is exactly how sometimes we can learn more from the SHG images than from fluorescence images. The perfect match of the SHG and TPEF images of compound $\mathbf{4}$ point towards an omnipresence in mitochondria, more specifically, a well-ordered, non-centrosymmetrically arranged presence, probably in the mitochondrial membranes. While we are not yet capable of determining whether these are inner or outer membranes, the SHG microscopy would suggest the outer membranes, we believe that the elaborately folded patterns of the inner membranes are less likely to lead to strong SHG signal and that SHG microscopy is therefore a guide towards additional structural information. The results from SHG imaging indicate that the staining mechanism will be different for the different dyes. Effects on mitochondrial activity were not investigated, but these might be able to shed light on these different mechanisms.

Setting compound $\mathbf{4}$, being also the smallest of the six selected markers, aside, we can divide the remaining compounds into two groups based on dimensionality or molecular symmetry of the dyes: the 1D (compounds 5, 7 and 9) and the 2D dyes (6 and 8). The 1D compounds reveal plasma membranes and a certain amount of vesicles, possibly large exosomes, as the cells might want to excrete the invading molecules. This can also explain why SHG only shows up when the fluorescence is already in place: the dyes target the mitochondria in the first place, after which the excess dye finds its way to the membranes, or stays there and reorients to achieve a highly ordered array of molecules. Compound 7 will have more trouble organizing itself inside the membranes as it is less planar than compounds $\mathbf{5}$ and $\mathbf{9}$, explaining why it only appears when the membranes loosen up. The reason why compound $\mathbf{4}$ does not show up in plasma membranes could be a high "solubility" of this dye in the mitochondria, or a poor ordering of the dye inside the plasma membrane.

The 2D compounds, although showing similar hydrophobicity, both behave differently compared to the $1 \mathrm{D}$ compounds and to each other. Compound 6 targets the nucleus in fluorescence, but probably invades the membranes the way the 1D compounds do. Compound $\mathbf{8}$ shows its fluorescence in the mitochondria, just like the $1 \mathrm{D}$ dyes, but it never exhibits SHG. Note that compound $\mathbf{8}$ is the $2 \mathrm{D}$ version of compound $\mathbf{7}$, and hence has two sites breaking the planarity of the molecule, which might completely prevent the molecules from finding a noncentrosymmetrical arrangement in the membranes, even when they start to lose their structural integrity.

It is interesting to compare the relative signal strength of the TPEF and the SHG in one location. The fluorescence signal coinciding with the SHG is very weak, sometimes nearly invisible due to the strength of the signal in the more concentrated parts of the sample. The SHG signal looks brighter because of the low background, but this difference is also explained by the coherent nature of SHG. While the incoherent TPEF signal scales linearly with the number of molecules in one pixel, the coherent SHG signal displays a quadratic dependence with respect to the number of molecules. ${ }^{1}$ This illustrates an extra asset in the power of SHG. The excess molecules can enter membranes, align with each other, and produce SHG with a much higher intensity and contrast than the TPEF signal. The molecules possibly even target membranes of organelles at random inside the cells, but so far, we are unable to defeat the diffraction limit using SHG microscopy to visualize the (membranes of) smaller organelles.

\section{Conclusions}

The second- and third-order nonlinear optical properties of a series of mono- and disubstituted carbazole-based derivatives have been determined by femtosecond frequency-resolved hyperRayleigh scattering and by two-photon excited fluorescence. These properties could be rationalized in terms of the electron acceptor strength of the substitutions on the donating carbazole moiety, in conjunction with two-photon resonance enhancement. The disubstituted carbazole derivatives show substantial twodimensional response, as seen from the low depolarization ratio 
and the derived sizeable off-diagonal tensor component for the first hyperpolarizability. These second-order nonlinear optical properties indicate that these carbazole-derived mono- and disubstituted chromophores are well-suited for combined SHG and multiphoton fluorescence microscopy.

In particular, it is shown that for all but the weakest acceptor substituents (pyridine and nitrophenyl), a second-order nonlinear response is observed at the second-harmonic wavelength (400 or $405 \mathrm{~nm}$ ) of the generic femtosecond laser system used in all multiphoton microscopy setups (a Ti:sapphire laser generating femtosecond pulses at about 800 or $810 \mathrm{~nm}$ ), in addition to multiphoton fluorescence that is conveniently separated and shifted to longer wavelengths than this secondharmonic wavelength. This means that for all the carbazole derivatives with the stronger acceptors, the second-order signal for SHG imaging (at $405 \mathrm{~nm}$ exactly) is spectrally nicely isolated from the longer-wavelength fluorescence used for high-resolution multiphoton imaging. No additional optics or algorithms are needed for the detection of SHG in the forward and TPEF in the backward direction. A simple color image can render the location of the chromophore (TPEF, longer wavelength) in combination with the structural information ( $\mathrm{SHG}$, second-harmonic wavelength, $405 \mathrm{~nm})$.

The potential of this class of chromophores for combined SHG and TPEF imaging has been demonstrated in two different human cell lines. In this pilot study, relatively high concentrations were used, but toxicity tests showed that this was justifiable for cultured cells. A well-considered selection of the compounds was tested for cellular NLO microscopy near $810 \mathrm{~nm}$. From this selection, all dyes can be used as selective markers for (multiplephoton) fluorescence, and compound $\mathbf{4}$ localizes identically in SHG, selectively staining the mitochondria in both channels. Compound $\mathbf{8}$ was proven not to exhibit SHG inside cells, and the other four selected compounds are visible in membranes shortly after the fluorescence is settled (or saturated), or specifically for compound 7, at the onset of visible cell death. From these results we can conclude that, even though the fluorescence seems to be identical for all compounds, the staining mechanism or exact target must be different for at least compound 4 . This confirms the power of SHG microscopy and the complementary information that can be achieved by it. SHG was confirmed by different imaging techniques, either based on the zero lifetime of the SHG signal or the sensitivity to the orientation of the laser polarization.

In this study, we have proven that compound $\mathbf{4}$ has substantial potential as a selective marker for mitochondria in combined TPEF and SHG microscopy. Compound $\mathbf{4}$ is a linear dipole with a considerable dynamic $\beta_{\mathrm{HRS}, 800}$, low toxicity for cell lines and good solubility in polar solvents. The fluorescence is strong and selective, as is the SHG at the wavelengths we used. The absorption and emission spectra are suitable for combined TPEF and SHG imaging using excitation near $800 \mathrm{~nm}$, the optimal performance of many commercially available pulsed lasers, and a good wavelength for deep penetration in biological samples. To our knowledge, this is the first specific marker for mitochondria in SHG microscopy.

In the near future, we will test the applicability of the markers in tissue, as well as in live animals, because an important application of nonlinear microscopy is to achieve deeper penetration depths. An additional research track would be to synthesize functionalized versions of the dyes for further study of intracellular structures and protein complexes.

\section{Acknowledgements}

E. D. M. would like to thank the Institute for Nanoscale Physics and Chemistry (INPAC) for her PhD fellowship, the financial support of the InterUniversity Attraction Poles (IAP p6/27) program and the Research Foundation Flanders (FWO) for her mobility grant. The authors of the Chinese Academy of Sciences thank the NSFC of China (No. 20702056, 50973126) and the National Basic Research Program of China (2010CB934103) for funding. S. V. C. thanks the agency for Innovation by Science and Technology (IWT) for his PhD fellowship. This work is also supported by the Spanish government grant TEC2009-09698. S. P. and P. L. A. acknowledge the Laserlab-Europe Cont (JRA4: Optobio 212025) and the Photonics for Life networks of excellence. This research has been partially supported by Fundació Cellex Barcelona and partially conducted at ICFO's SuperResolution Light Nanoscopy Facility (SLN@ICFO). J. M. T. is a senior postdoctoral researcher of the Research Foundation Flanders (FWO). The authors thank Dr Angels Serra (IDIBELL, Spain), Dr Claudia Nieva Boza (ICFO, Spain), and Dr Hideaki Mizuno (K.U.Leuven, Belgium) for providing the cell lines.

\section{References}

1 J. E. Reeve, H. L. Anderson and K. Clays, Phys. Chem. Chem. Phys, 2010, 12, 13484-14498.

2 H.-P. Zhou, Y.-P. Tian, J.-Y. Wu, J.-Z. Zhang, D.-M. Li, Y.-M. Zhu, Z.-J. Hu, X.-T. Tao, M.-H. Jiang and Y. Xie, Eur. J. Inorg. Chem., 2005, 4976-4984.

3 J. E. Reeve, H. A. Collins, K. De Mey, M. M. Kohl, K. J. Thorley, O. Paulsen, K. Clays and H. L. Anderson, J. Am. Chem. Soc., 2009, 131, 2758-2759.

4 M. J. Therien, Nature, 2009, 458, 716-717.

5 C. Barsu, R. Fortrie, K. Nowika, P. L. Baldeck, J.-C. Vial, A. Barsella, A. Fort, M. Hissler, Y. Bretonniere, O. Maury and C. Andraud, Chem. Commun., 2006, 4744-4746.

6 T. Verbiest, K. Clays and V. Rodriguez, Second-order Nonlinear Optical Characterization Techniques: An Introduction; Taylor and Francis, CRC Press, Boca Raton, FL, 2009.

7 K. Clays, R. J. Docherty, J. Fielden, M. Helliwell, J. Raftery, R. Shaw, C. A. Swanson and B. J. Coe, Proc. Soc. Photo. Opt. Instrum. Eng., 2010, 781713.

8 M. G. Kuzyk and C. W. Dirk, Characterization Techniques and Tabulations for Organic Nonlinear Optical Materials, Marcel Dekker, New York, 1998.

9 P. A. Sullivan and L. R Dalton, Acc. Chem. Res., 2010, 43, 10-18.

10 C. Barsu, R. Cheaib, S. Chambert, Y. Queneau, O. Maury, D. Cottet, H. Wege, J. Douady, Y. Bretonniere and C. Andraud, Org. Biomol. Chem., 2010, 8, 142-150.

11 C. Boutton, K. Clays, A. Persoons, T. Wada and H. Sasabe, Chem. Phys. Lett., 1998, 286, 101-106.

12 X. J. Feng, P. L. Wu, F. Bolze, H. W. C. Leung, K. F. Li, N. K. Mak, D. W. J. Kwong, J.-F. Nicoud, K. W. Cheah and M. S. Wong, Org. Lett., 2010, 12, 2194-2197.

13 J.-F. Xing, W.-Q. Chen, J. Gu, X.-Z. Dong, N. Takeyasu, T. Tanaka, X.-M. Duan and S. Kawata, J. Mater. Chem., 2007, 17, 1433-1438.

14 J. Gu, W. Yulan, W.-Q. Chen, X.-Z. Dong, X.-M. Duan and S. Kawata, New J. Chem., 2007, 31, 63-68.

15 M.-L. Zheng, K. Fujita, W.-Q. Chen, N. I. Smith, X.-M. Duan and S. Kawata, ChemBioChem, 2011, 12, 52-55.

16 G. Olbrechts, R. Strobbe, K. Clays and A. Persoons, Rev. Sci. Instrum., 1998, 69, 2233-2241.

17 B. F. Levine and C. G. Bethea, J. Chem. Phys., 1975, 63, 2666-2682. 
18 K. D. Singer and A. F. Garito, J. Chem. Phys., 1981, 75, 3572-3580.

19 K. Clays, K. Wostyn, K. Binnemans and A. Persoons, Rev. Sci. Instrum., 2001, 72, 3215-3220.

20 C. Xu and W. W. Webb, J. Opt. Soc. Am. B, 1996, 13, 481-491.

21 K. N. Anisha Thayil, E. J. Gualda, S. Psilodimitrakopoulos, I. G. Cormack, I. Amat-Roldán, M. Mathew, D. Artigas and P. Loza-Alvarez, J. Microsc., 2008, 230, 70-75.

22 J.-L. Brédas, C. Adant, P. Tackx and A. Persoons, Chem. Rev., 1994, 94, 243-278.

23 J. L. Oudar and D. S. Chemla, J. Chem. Phys., 1977, 66, 2664-2668.

24 J. L. Oudar, Chem. Phys., 1977, 67, 446-457.

25 X.-M. Duan, S. Okada, A. Watanabe, M. Matsuda, K. Clays, A. Persoons and H. Nakanishi, Proc. Soc. Photo. Opt. Instrum. Eng., 1994, 2143, 41-51.

26 J. R. Lakowicz, Principles of Fluorescence Spectroscopy, Springer Science, Berlin, 2007.

27 S. Psilodimitrakopoulos, S. I. Santos, I. Amat-Roldan, A. K. Thayil, D. Artigas and P. Loza-Alvarez, J. Biomed. Opt., 2009, 14(1), 014001.

28 C. R. Cantor and P. R. Schimmel, Biophysical Chemistry: Part II Techniques for the study of biological structure and function, W.H. Freeman, San Francisco, CA, 1980.

29 E. De Meulenaere, S. Van Cleuvenbergen, S. Psilodimitrakopoulos, J. Vanderleyden, P. Loza-Alvarez and K. Clays, Proc. SPIE-Int. Soc. Opt. Eng., 2011, 80861B.
30 D. Dumas, C. Henrionnet, S. Hupont, E. Werkmeister, J. F. Stoltz, A. Pinzano and P. Gillet, Bio-Med. Mater. Eng., 2010, 20, 183188.

31 S. Psilodimitrakopoulos, V. Petegnief, G. Soria, I. Amat-Roldan, D. Artigas, A. M. Planas and P. Loza-Alvarez, Opt. Express, 2009, 17, 14418-14425.

32 P. J. Campagnola, M. D. Wei, A. Lewis and L. M. Loew, Biophys. J., 1999, 77, 3341-334.

33 L. Moreaux, O. Sandre, M. Blanchard-Desce and J. Mertz, Opt. Lett., 2000, 25, 320-322.

34 T. Petralli-Mallow, T. M. Wong, J. D. Byers, H. I. Yee and J. M. Hicks, J. Phys. Chem., 1993, 97, 1383-1388.

35 J. D. Byers, H. I. Yee and J. M. Hicks, J. Chem. Phys., 1994, 101, 6233-6241.

36 V. K. Valev, A. V. Silhanek, N. Smisdom, B. De Clercq, W. Gillijns, O. A. Aktsipetrov, M. Ameloot, V. V. Moshchalkov and T. Verbiest, Opt. Express, 2010, 18, 8286-8293.

37 L. V. Johnson, M. L. Walsh and L. B. Chen, Proc. Natl. Acad. Sci. U. S. A., 1980, 77, 990-994.

38 C.-C. Chang, I.-C. Kuo, I.-F. Ling, C.-T. Chen, H.-C. Chen, P.-J. Lou, J.-J. Lin and T.-C. Chang, Anal. Chem., 2004, 76, 44904494.

39 C.-C. Chang, J.-F. Chu, F.-J. Kao, Y.-C. Chiu, P.-J. Lou, H.-C. Chen and T.-C. Chang, Anal. Chem., 2006, 78, 2810-2815. 